\title{
Respecto al artículo "El mosquito del dengue en la Ciudad de México. Invasión incipiente de Aedes aegypti y sus potenciales riesgos"
}

\author{
Regarding the article "Aedes aegypti, the dengue fever mosquito in Mexico City. Early \\ invasion and its potential risks" \\ Antonio Reyna-Sevilla* \\ Instituto Mexicano del Seguro Social, Dirección de Prestaciones Médicas, Coordinación de Planeación en Salud, Ciudad de México, México
}

Después de leer atentamente el artículo de María Dolores Mejía Guevara et al., ${ }^{1}$ en el que se invita oportunamente a controlar el riesgo de enfermedades transmitidas por vector en la Ciudad de México, advertí un aspecto metodológico omitido: la indicación de si los sitios donde se colocaron las ovitrampas fueron elegidos aleatoriamente o cuáles fueron los criterios establecidos para la selección.

La respuesta resulta especialmente importante porque en determinados lugares de la Ciudad de México - según sus condiciones geográficas como altitud, régimen pluviométrico anual, tipo de vegetación (ruderal), cobertura vegetal $\left(\mathrm{km}^{2}\right)$, porcentaje de humedad relativa, densidad poblacional, entre otrases posible que exista una mayor o menor concentración de huevecillos de Aedes aegypti, lo cual pudo haber sesgado no solo la recolección y el análisis de los datos, sino también los resultados.
Sin embargo, considerando que los autores utilizaron datos georreferenciados junto con la técnica de superposición de mapas vectoriales, ${ }^{2,3}$ es probable que pudieran emplear otras herramientas como puntos aleatorios en la extensión (área de estudio), puntos aleatorios en la capa (de información) o puntos aleatorios dentro de los polígonos (delimitación de alcaldías), disponibles en los sistemas de información geográfica para evitar posibles sesgos en los resultados, que, sin duda, deben ser considerados.

\section{Bibliografía}

1. Mejía-Guevara MD, Correa-Morales F, González-Acosta C, Dávalos-Becerril E, Peralta-Rodríguez JL, Martínez-Gaona A, et al. El mosquito del dengue en la Ciudad de México. Invasión incipiente de Aedes aegypti y sus potenciales riesgos. Gac Med Mex. 2020;156:388-395.

2. Buzai G. Análisis espacial en geografía de la salud. Resoluciones con sistemas de información geográfica. Argentina: Lugar Editorial; 2015.

3. Del Bosque I, Fernández C, Forero L, Pérez E. Los sistemas de información geográfica y la investigación en ciencias humanas y sociales. España: Confederación Española de Centros de Estudios Locales; 2012.
Gac Med Mex. 2021:157:333

Disponible en PubMed www.gacetamedicademexico.com CC BY-NC-ND (http://creativecommons.org/licenses/by-nc-nd/4.0/). 